\title{
Técnicas difusas para la evaluación de impactos ambientales en el transporte de hidrocarburos en Colombia
}

\section{Fuzzy techniques for environmental impact assessment in hydrocarbons}

\author{
transportation in Colombia
}

\section{Germán Yesid Luque Díaz ${ }^{10}$, Laura Cristina Ramírez Salinas ${ }^{2}$ Mauricio Andrés Ruíz Ochoa $^{3}$}

Fecha de recepción: 20 Septiembre 2019

Fecha de aceptación: 05 de Febrero de 2020

Cómo citar: Luque-Díaz., G.Y., Ramírez-Salinas., L.C., y Ruíz-Ochoa., M.A., (2020). Técnicas difusas para la evaluación de impactos ambientales en el transporte de hidrocarburos en Colombia. Tecnura, 24(64). 48-65 https://doi.org/10.14483/22487638.15762

\section{RESUMEN}

Objetivo: El propósito del trabajo fue aplicar técnicas difusas para que sean usadas como metodologías para reducir la incertidumbre y la subjetividad.

Metodología: Para este artículo, debido a la magnitud de los impactos ambientales, solo se presentan los resultados de aplicar las técnicas difusas sobre la acción susceptible de producir impactos (ASPI) desmonte y descapote, perteneciente a la etapa de construcción del proyecto construcción y operación de la interconexión Caño Limón-Guafita Cruce Subfluvial Río Arauca. Los resultados fueron validados a través de la metodología de Conesa.
Resultados: Se encontraron diez impactos que afectaron los medios físico, biótico y socioeconómico y cultural; y los componentes geología y geomorfología, suelos, hidrogeología, agua, atmósfera, flora, fauna, y aspectos arqueológicos. Al incorporar los conjuntos de números difusos, estos se agruparon en cuatro grandes categorías: muy posiblemente moderado, con tres impactos; posiblemente severo, con dos impactos; posiblemente moderado, con tres impactos; y poco posiblemente severo, con dos impactos. Con la metodología propuesta, el $40 \%$ de ellos resultaron ser severos (poco posiblemente y posiblemente), y el porcentaje restante, moderados (posiblemente y muy posiblemente); mientras que con Conesa no

Ingeniero Ambiental. Independiente. Bucaramanga, Colombia. Contacto: germanluquediaz@gmail.com

ORCID: https://orcid.org/0000-0003-4773-3300

Ingeniera Ambiental. Independiente. Bucaramanga, Colombia. Contacto: goritoramirez@gmail.com

ORCID: https://orcid.org/0000-0002-9768-0668

Ingeniero Ambiental, magíster en Ingeniería - Recursos Hidráulicos, doctor en Ingeniería. Docente de las Unidades Tecnológicas de

Santander. Bucaramanga, Colombia. Contacto: mruiz@correo.uts.edu.co

ORCID: https://orcid.org/0000-0002-8374-4165 
se presentaron impactos severos, los impactos moderados representaron el $30 \%$, y en su gran mayoría, fueron valorados como irrelevantes.

Conclusiones: La metodología propuesta resalta las debilidades de la metodología convencional, debido a la poca subjetividad al momento de valorar los impactos, lo cual facilita la toma de decisiones. Por tanto, esta metodología modela con mayor exactitud la interacción proyecto/ ambiente ajustada a escenarios más reales.

Financiamiento: Unidades Tecnológicas de Santander.

Palabras clave: evaluación de impacto ambiental; números difusos; técnicas difusas.

\section{ABSTRACT}

Objective: The objective of the work was to apply fuzzy techniques to be used as one of these methodologies to reduce uncertainty and subjectivity.

Methodology: For this article, due to the magnitude of the environmental impacts, we only present the results of applying the fuzzy techniques on the action susceptible to produce impacts (ASPI) "clearing and stripping", belonging to the construction stage of the Project Construction and Operation of the interconnection Caño Limón-Guafita Crossing Sub-River Arauca. The results were validated using Conesa's methodology.
Results: Ten impacts were found that affected the physical, biotic and socioeconomic and cultural environments; and the components geology and geomorphology, soils, hydrogeology, water, atmosphere, flora, fauna, and archaeological aspects. By incorporating the fuzzy sets numbers, they are grouped into four broad categories: very possibly moderate with 3 impacts; possibly severe with 2 impacts; possibly moderate with 3 impacts; and little possibly severe with 2 impacts. With the proposed methodology, $40 \%$ turned out to be severe (little possibly and possibly), and the remaining percentage, moderate (possibly and very possibly); while with Conesa there are no severe impacts, moderate impacts represented $30 \%$, and the great majority were valued as irrelevant.

Conclusions: The proposed methodology highlights the weaknesses of conventional methodology, due to the lack of subjectivity when assessing impacts, which facilitates decision-making. Therefore, this methodology models more accurately the project-environment interaction adjusted to more real-life scenarios.

Financing: Unidades Tecnológicas de Santander.

Keywords: environmental impact assessment; fuzzy numbers; fuzzy arithmetic.

\section{INTRODUCCIÓN}

En los últimos años el sector petrolero ha perdido fuerza por su bajo costo en el mercado, pero es innegable que este constituye hoy en día uno de los principales elementos del PIB de Colombia, y su uso lo convierte en una fuente de energía importante (Corficolombiana, 2018; Malagón, Montoya y Ruiz, 2016).
No obstante, esta industria es una de las que más genera impactos ambientales en la biodiversidad a nivel global y local (Avellaneda, 1990; Bravo, 2005; Correa, Marulanda y Panesso, 2016; Parada et al., 2016; Rojas, Duacuara y Moreno, 2015). Asimismo, el transporte de crudo, aunque se encuentra fiscalizado desde los nodos de entrada hasta los nodos de salida incluyendo, entre otros, la tubería, las unidades de bombeo, 
las estaciones de medición, los sistemas de control y los tanques que se usan para la operación del sistema de transporte, también generan perturbaciones sobre el ambiente (Avellaneda, 1990; Collazos, Esquivel y Paz, 2019; Guerrero, 2018).

La evaluación de impacto ambiental (EIA), como herramienta para identificar tipo, magnitud y cambios potenciales causados sobre el ambiente por las actividades de los proyectos (Bailey, 1997; Toro, Requena y Zamorano, 2010), solo tomó importancia después de la Conferencia de Estocolmo en 1972, a través de la Ley Nacional de Política Ambiental (NEPA, por su sigla en inglés) (Bear, 2003; Luther, 2008), lo cual se consolidó con la creación del Programa de las Naciones Unidas para el Medio Ambiente (PNUMA) (Naciones Unidas, 2012). El objetivo de la EIA fue crear conciencia mundial acerca de los problemas ambientales generados por las grandes obras de ingeniería que se estaban desarrollando en Estados Unidos (Bailey, 1997; Luther, 2008; Naciones Unidas, 2012; Toro, Requena y Zamorano, 2010).

Actualmente, la ElA se considera un proceso que busca de manera anticipada prevenir/mitigar los impactos negativos derivados de las actividades del proyecto, de tal forma que se puedan implementar medidas de control para beneficio del ambiente (Marchevsky, Giubergia y Ponce, 2018; Toro, Requena y Zamorano, 2010; Toro, Duarte, Requena y Zamorano, 2012; Toro, Martínez y Arrieta, 2013). En Colombia las metodologías de EIA aplicadas, de manera general y crítica, se presentan en Toro, Requena y Zamorano (2010); Toro, Duarte, Requena y Zamorano (2012), y Toro, Martínez y Arrieta (2013); para proyectos de infraestructura, en Viloria, Cadavid y Awad (2018); mientras que para el sector de hidrocarburos, se tienen Lozano (2014) y Guerrero (2018), y en todas se evidencia el grado de subjetividad asociado a la valoración de los impactos.

Frente a lo anterior, Luque y Ramírez (2016), con énfasis en el transporte y conducción de hidrocarburos, mencionan que en la realización de la EIA independientemente del método se presentan dos dificultades inherentes a la naturaleza de dichas evaluaciones, las cuales están relacionadas con: a) la forma en que el proyecto repercutirá sobre el entorno, dado que la EIA es una predicción $y$, por tanto, tiene una incertidumbre asociada, y b) el entono es muy complejo y no se puede describir con un único modelo. Además, aunque cada factor ambiental puede ser analizado por separado, estos son muy diferentes entre sí, y lo más complejo es poder agregar la información parcial de cada uno de ellos, con el fin de obtener un análisis global del entorno (Duarte, 2000; Duarte, Requena y Rosario, 2007; Morrillas, 2006; Villareal y Arango, 2014).

Esta situación se acentúa aún más si, como es usual, la evaluación de cada factor se lleva a cabo por un experto (o un grupo de expertos) diferente (Morrillas, 2006; Toro, Requena y Zamorano, 2010; Toro, Duarte, Requena y Zamorano, 2012; Toro, Martínez y Arrieta, 2013; Viloria, Cadavid y Awad, 2018). A su vez, algunas de las variables involucradas en la EIA son de tipo numérico (cuantitativo), mientras que otras son de tipo lingüístico (cualitativo). En este contexto Luque y Ramírez (2016) argumentan que las variables empleadas en las EIA se refieren a conceptos sin fundamentos teóricos, los cuales se realizan a partir de variables lingüísticas (Zadeh, 1975; Morrillas, 2006). Por ejemplo, el valor que se le da a la importancia de un impacto se ajusta 
a criterios de irrelevante, moderado, severo o crítico, que corresponden a simples etiquetas semánticas. Casi que obliga a modelar el entorno como un conjunto de factores ambientales que sean relevantes, representativos y fácilmente analizables. Así, se requiere un modelo matemático que combine ambos tipos de variables de forma coherente, y disminuya la subjetividad (Duarte, Requena y Rosario, 2007; Dutta, Boruah y Ali, 2011; Villareal y Arango, 2014).

Para disminuir la subjetividad existen las técnicas difusas que son una metodología precisa, que evidencian de manera más real cómo influirán sobre el entorno las actividades del proyecto, una vez se realicen. Estas emplean el cálculo de un índice de importancia para diferenciar una clasificación por intervalos (Duarte, 2000; Luque y Ramírez, 2016; Morrillas, 2006). Por ejemplo, si al calcular la importancia de dos impactos, resulta que para uno es 49 y para el otro es 50, al momento de decidir se tendría una dualidad planteada en el siguiente interrogante: ¿Es tal la diferencia entre un impacto y otro como para clasificar al primero como moderado y el segundo como severo?, es decir, la incertidumbre estaría presente.

Para responder a lo anterior, las técnicas difusas a partir de las variables lingüísticas (Zadeh, 1975; Morrillas, 2006) definen rangos de clasificación de los impactos representados por conjuntos difusos, dentro de los cuales se incluyen los números difusos (Dutta, Boruah y Ali, 2011; Villareal y Arango, 2014), los cuales permiten modelar adecuadamente los valores numéricos en los que exista dicha incertidumbre (Dutta, Boruah y Ali, 2011), lo que permite tomar medidas correctivas más ajustadas a la realidad del proyecto. A su vez, tal como lo men- ciona Duarte (2000), la EIA mediante técnicas difusas se basa en las metodologías convencionales, por lo que se considera una extensión de estas.

En este trabajo se propone una metodología cuantitativa de EIA para el transporte de hidrocarburos en Colombia, con el fin de ayudar en la toma de decisiones frente al desarrollo de las actividades de los proyectos.

\section{METODOLOGÍA}

\section{Métodos}

Se propone una metodología para evaluar los impactos producidos por el transporte de hidrocarburos en Colombia, la cual se ajusta a los términos de referencia y a la normatividad vigente. La información para el trabajo fue suministrada por la Autoridad Nacional de Licencias Ambientales (ANLA).

Se tomó como base el trabajo de pregrado de Luque y Ramírez (2016), y como caso práctico de aplicación para este artículo, se seleccionó la acción susceptible de producir impactos (ASPI), desmonte y descapote, pertenecientes al Proyecto de Construcción y Operación de la Interconexión Caño Limón-Guafita Cruce Subfluvial Río Arauca. Se escogió esta ASPI porque es una actividad que representa la remoción de la capa superficial del terreno con medios mecánicos, lo cual afecta la dinámica del ciclo hidrológico (Poveda y Mesa, 1995) en los diferentes ecosistemas.

La identificación de los impactos ambientales para el ASPI seleccionado se realizó mediante el diagrama de procesos y el método matricial, ambos basados en la secuencia entrada/proceso/salida/impacto, tal como lo describe Arboleda (2008). 
Se aplicó la metodología de ElA basada en técnicas difusas y sus resultados se validaron con los obtenidos con la metodología de Conesa aplicada por los expertos del proyecto en mención.

\section{Técnicas difusas para la valoración del im- pacto}

Para evaluar los impactos mediante el método de técnicas difusas se siguieron los pasos dados por Duarte (2000) y Duarte, requena y Rosario (2007), donde las palabras de entrada corresponden a los criterios a evaluar; a estos se les dio una interpretación numérica difusa, lo cual permitió transformarlos en conjuntos difusos de entrada. Para cada criterio se asignó un peso que se encuentra establecido por la metodología de Conesa (tabla 1).

Este se ajustó de acuerdo con la metodología propuesta, para una función de razonamiento aproximado (fra) mediante la ecuación (1).

$$
\text { fra: } y=\sum_{i=1}^{n} f_{i} w_{i} g_{i}\left(x_{i}\right)+\sum_{i=1}^{n}\left(1-f_{i}\right) w_{i} g_{i}\left(1-x_{i}\right)
$$

Para la cual, si la salida (y) es monótonamente creciente a la entrada $i$, es porque se dio un valor $f_{i}=1$; si, por el contrario, la salida $(y)$ es monótonamente decreciente a la entrada $i$, se obtiene un valor $f_{i}=0$. La variable $w_{i}$ es el peso asociado a la entrada i, la suma de todos los pesos de entrada debe ser 1 . La función $g_{i}\left(x_{i}\right)$ asignada fue $g_{i}\left(x_{i}\right)=\llbracket\left(x_{i} \rrbracket^{\wedge}\left(\theta_{i}\right)\right)$; donde $\theta_{i}$ es un exponente seleccionado de acuerdo con las necesidades de la evaluación, para valores de $\theta_{i}>1$ permiten subvalorar los valores bajos de $x_{i}$, y para valores de $\theta_{i}>1$ permiten sobrevalorar los valores bajos de $x_{i}$. Se escogió por defecto un valor de $\theta_{i}=2$.

Tabla 1: Criterios a evaluar por parte de la metodología propuesta y conjuntos difusos de entrada

\begin{tabular}{|c|c|c|c|c|}
\hline Criterios & $\begin{array}{l}\text { Escala de } \\
\text { valoración }\end{array}$ & Descripción & Peso & Conjuntos difusos \\
\hline Naturaleza (NA) & $\begin{array}{l}\text { Positivo (+) } \\
\text { Negativo (-) }\end{array}$ & $\begin{array}{l}\text { Carácter del impacto resultado de la } \\
\text { acción sobre el factor ambiental. }\end{array}$ & NA & NA \\
\hline Extensión (EX) & $\begin{array}{l}\text { Puntual Pt) } \\
\text { Parcial (Pr) } \\
\text { Extenso (Ext) } \\
\text { Total }(\mathrm{T}) \\
\end{array}$ & $\begin{array}{l}\text { Área de influencia teórica del impacto } \\
\text { en relación con el entorno del proyecto } \\
\text { en que se sitúa el factor. }\end{array}$ & $2 / 15$ & $\begin{array}{l}{\left[\begin{array}{llll}0,00 & 0,00 & 0,14 & 0,29\end{array}\right]} \\
{\left[\begin{array}{llll}0,14 & 0,29 & 0,43 & 0,57\end{array}\right]} \\
{\left[\begin{array}{llll}0,43 & 0,57 & 0,71 & 0,86\end{array}\right]} \\
{\left[\begin{array}{llll}0,71 & 0,86 & 1,00 & 1,00\end{array}\right]} \\
\end{array}$ \\
\hline Extensión crítica (EC) & Extensión crítica & $\begin{array}{l}\text { Cuando el efecto sea puntual o no, se } \\
\text { produzca en un lugar crucial o crítico, } \\
\text { será un impacto de ubicación crítica } \\
\text { aumentando el grado de incidencia de } \\
\text { la extensión. }\end{array}$ & $1 / 15$ & {$\left[\begin{array}{llllll}0,00 & 0,00 & 1,00 & 1,00\end{array}\right.$} \\
\hline Persistencia (PE) & $\begin{array}{c}\text { Fugaz }(\mathrm{Fz}) \\
\text { Temporal }(\mathrm{Tm}) \\
\text { Persistente }(\mathrm{Ps}) \\
\text { Permanente }(\mathrm{Pm}) \text {. }\end{array}$ & $\begin{array}{l}\text { Tiempo que permanecería el efecto } \\
\text { desde su aparición y a partir del cual } \\
\text { el factor afectado retornaría a las } \\
\text { condiciones iniciales. }\end{array}$ & $1 / 15$ & $\begin{array}{l}{\left[\begin{array}{llll}0,00 & 0,00 & 0,02 & 0,05\end{array}\right]} \\
{\left[\begin{array}{llll}0,02 & 0,05 & 0,29 & 0,37\end{array}\right]} \\
{\left[\begin{array}{llll}0,29 & 0,37 & 0,46 & 0,54\end{array}\right]} \\
{\left[\begin{array}{llll}0.46 & 0.54 & 1,00 & 1,00\end{array}\right]}\end{array}$ \\
\hline
\end{tabular}




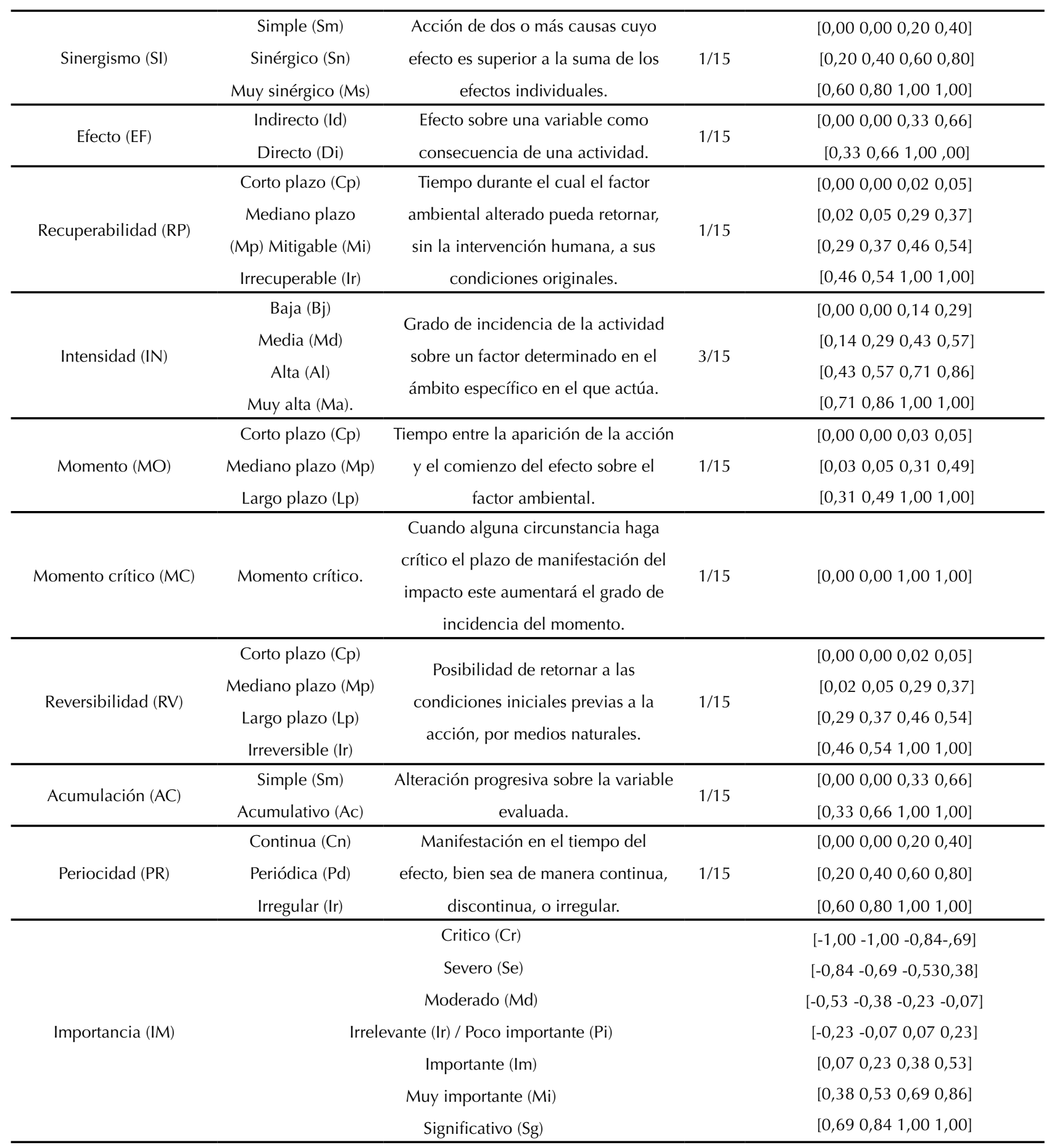

Fuente: Luque y Ramírez (2016).

Las técnicas difusas consisten en la representación de indicadores y variables por medio de números difusos, los cuales no poseen una estructura algebraica de grupo (Duarte 2000; Duarte, Requena y
Rosario, 2007; Dutta, Boruah y Ali, 2011). Sin embargo, se estableció un principio de extensión mediante el cual se modificaron las funciones del modelo para operar con aritmética difusa (Villareal y Arango, 2014). 


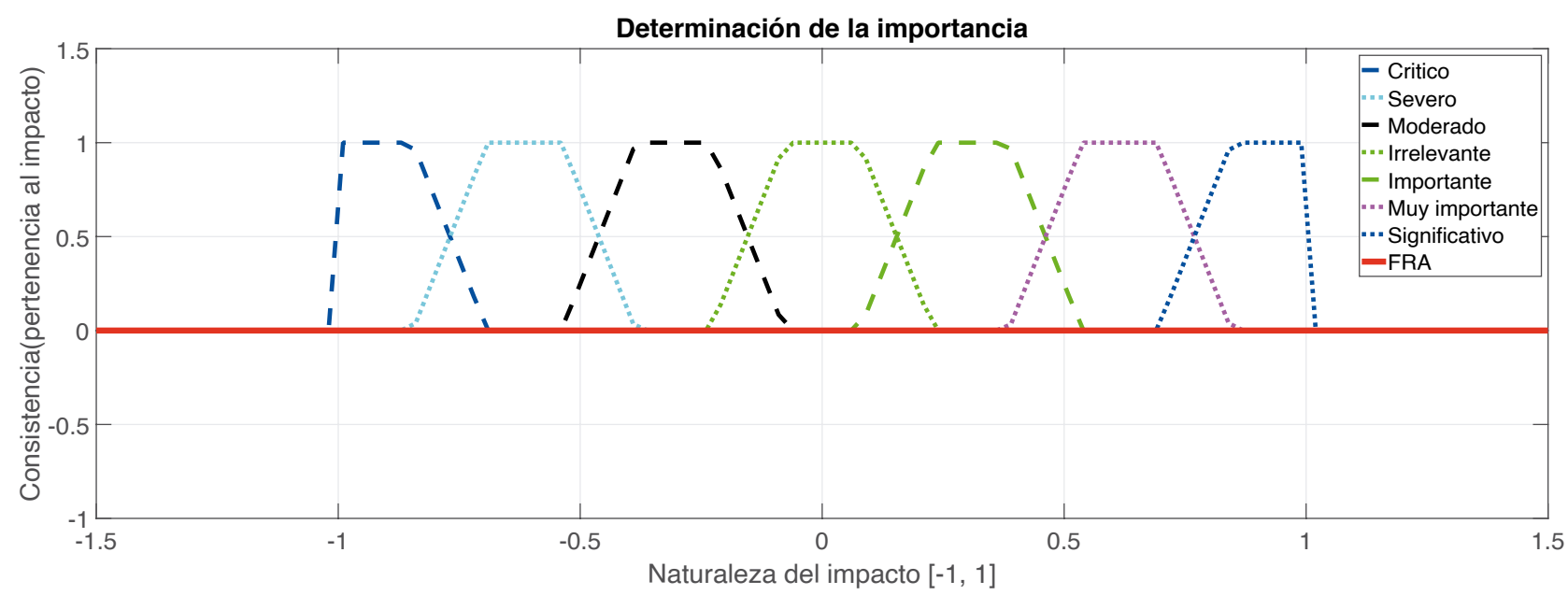

Figura 1. Ejemplo de ejecución Guide (MATLAB)

Fuente: Luque y Ramírez (2016).

Se usó una función fra, programada en MATLAB (figura 1), para obtener en la salida un número difuso que representará la intensidad de la importancia de cada impacto sobre determinado factor ambiental.

\section{RESULTADOS}

\section{Identificación de impactos}

En general, Luque y Ramírez (2016) para todo el proyecto identificaron 73 impactos distribuidos en cuatro etapas del proyecto, así: preconstrucción, un impacto asociado al componente dimensión económica; construcción, 67 impactos distribuidos en los componentes, geología y geomorfología, suelo, hidrología, calidad del agua, uso del agua, hidrogeología, geotecnia, paisaje, calidad del aire, ruido, fauna, flora, dimensión económica y dimensión político-organizativa; operación, 3 impactos para los componentes dimensión económica y dimensión político-organizativa; y abandono técnico, 2 impactos en la dimensión económica.

Como caso práctico de aplicación de la metodología propuesta, en este artículo solo se presentan los impactos asociados al ASPI, desmonte y descapote de la etapa de construcción (tabla 2). Se encontraron 10 impactos que afectaron los medios físico, biótico y socioeconómico y cultural; y los componentes geología y geomorfología, suelos, hidrogeología, agua, atmósfera, flora, fauna y aspectos arqueológicos.

\section{Metodología de EIA propuesta}

El peso ponderado de cada criterio fue el establecido por la metodología de Conesa (tabla 1), este se ajustó a la fra, la cual es un número difuso trapezoidal definido para el intervalo $[0,1]$. Lo anterior, permitió definir con precisión la pertenencia o no, de un elemento al subconjunto de entrada (Duarte, Requena y Rosario, 2007; Dutta, Boruah y Ali, 2011). Los 
Tabla 2. Impactos ambientales asociados al ASPI, desmonte y descapote.

\begin{tabular}{|c|c|c|c|c|c|}
\hline$\frac{\mathscr{2}}{\stackrel{\Xi}{ \pm}}$ & $\overline{\bar{\alpha}}$ & 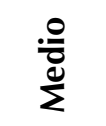 & $\begin{array}{l}\text { Componente } \\
\text { ambiental }\end{array}$ & Impacto ambiental & $\begin{array}{l}\text { ID del } \\
\text { impacto }\end{array}$ \\
\hline \multirow{10}{*}{ 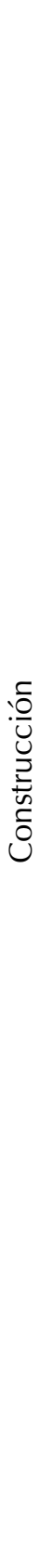 } & \multirow{10}{*}{ 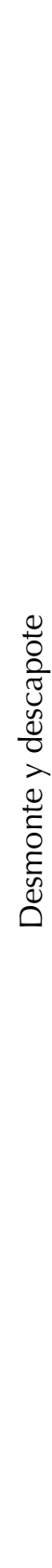 } & \multirow{8}{*}{$\frac{\stackrel{0}{n}}{\frac{n}{4}}$} & $\begin{array}{l}\text { Geología y } \\
\text { geomorfología }\end{array}$ & $\begin{array}{l}\text { Deterioro de las formas del } \\
\text { terreno. }\end{array}$ & DFT \\
\hline & & & Suelos & $\begin{array}{l}\text { Deterioro en las propiedades } \\
\text { físicas, químicas y biológicas del } \\
\text { suelo. }\end{array}$ & DPF \\
\hline & & & Hidrogeología & $\begin{array}{l}\text { Disminución en el volumen de } \\
\text { agua a infiltrarse. }\end{array}$ & DVA \\
\hline & & & Agua & $\begin{array}{l}\text { Deterioro de la propiedades físicas, } \\
\text { químicas y microbiológicas de las } \\
\text { aguas. }\end{array}$ & DPFa \\
\hline & & & \multirow{2}{*}{ Atmósfera } & Deterioro de la calidad del aire. & DCA \\
\hline & & & & $\begin{array}{l}\text { Aumento de los niveles de presión } \\
\text { sonora. }\end{array}$ & ANP \\
\hline & & & Flora & Pérdida de cobertura. & $\mathrm{PCO}$ \\
\hline & & & \multirow[b]{2}{*}{ Fauna } & $\begin{array}{l}\text { Disminución de las poblaciones de } \\
\text { fauna silvestre. }\end{array}$ & DPF \\
\hline & & $\stackrel{0}{: 0}$ & & $\begin{array}{l}\text { Pérdida de hábitat para fauna } \\
\text { terrestre. }\end{array}$ & PHF \\
\hline & & 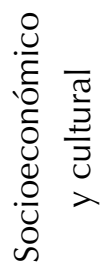 & $\begin{array}{l}\text { Aspectos } \\
\text { arqueológicos }\end{array}$ & $\begin{array}{l}\text { Pérdida del patrimonio } \\
\text { arqueológico. }\end{array}$ & PPA \\
\hline
\end{tabular}

Fuente: Luque y Ramírez (2016). 
números difusos trapezoidales fueron definidos como $T:\left(a_{1}, a_{2}, a_{3}, a_{4}\right)$, donde se debía cumplir que $T:\left(a_{1}>a_{2}>a_{3}>a_{4}\right)$, lo cual era una condición necesaria para poder operar aplicando aritmética difusa por el método de los $\alpha$-cortes (Dutta, Boruah y Ali, 2011).

Teniendo en cuenta lo mencionado por Duarte, Requena y Rosario (2007) y Dutta, Boruah y Ali (2011), los números difusos fueron definidos para los números trapezoidales de la forma $\mathrm{T}:\left[a_{1}+\left(a_{2}-a_{1}\right) \alpha, a_{4}-\left(a_{4}-a_{3}\right) \alpha\right] \quad \forall \alpha \in[0,1]$, donde $\alpha$ representa el corte con el eje $y$. Los $\alpha$-corte permitieron modelar por medio de dos puntos el comportamiento de la gráfica en el rango $[0,1]$.

Una vez se estableció la naturaleza del impacto, la importancia difusa se evaluó sobre el intervalo [-1, 1]. Es de resaltar que para el criterio MO, se encontró que la importancia del impacto es monótonamente decreciente, es decir, a medida que el momento crece la importancia del impacto disminuye. Esto fue tenido en cuenta para interpretar su probabilidad de pertenencia o no, al conjunto de importancia difusa (crítico, severo, moderado, irrelevante, poco importante, importante y muy importante); esto igualmente se asoció a las etiquetas muy posiblemente, posiblemente y poco posiblemente. En la tabla 3 se presenta la aproximación lingüística dada a cada impacto y la importancia difusa obtenida.

Para la aplicación de la técnica se requirieron variables de entrada (tabla 3), que son conjuntos (criterios para la evaluación del impacto) constituidos por subconjuntos definidos de forma más precisa. Estos subconjuntos de entrada fueron definidos como números difusos trapezoidales de dos tipos, cualitativos o cuantitativos, como lo expresan Duarte (2000) y Duarte, Requena y Rosario (2007).

Para los conjuntos difusos de entrada tipo cualitativos, la construcción de los conjuntos difusos se realizó según Duarte (2000), quien propuso que el universo de discurso debía distribuirse de forma equitativa para cada una de las variables (tabla 4).

Para el caso de las variables cuantitativas Duarte (2000) propuso que cuando se tengan puntos de cruce entre números difusos, se debe tomar el $25 \%$ de los datos del dominio sobre el universo de discurso para el menor subconjunto, el cual se adiciona y se resta al límite entre los subconjuntos para conformar la diferencia entre soporte y núcleo, con esto se estableció un equivalente a los datos más representativos de la etiqueta, a excepción del primer y último número difuso que no presenta una diferencia entre núcleo y soporte para el lado izquierdo y para el lado derecho del número, respectivamente. Por otra parte, para los criterios MO, PE, $R V$ y RP, al estar definidos en una escala de tiempo se pudieron expresar directamente bajo números difusos que representaran dichas escalas.

Para el caso del MO, este se construyó teniendo en cuenta las escalas de valoración definidas en la tabla 1, tomando como un límite superior los 25 años. Sin embargo, cualquier valor después de los 10 años fue representado con la etiqueta a largo plazo, pero se escogió un valor mucho mayor al dominio de a mediano plazo para que al representarlo como un número difuso más grande exprese el cambio en el tiempo con respecto a los demás subconjuntos del criterio. 
Tabla 3. Variables lingüísticas para la evaluación de la metodología

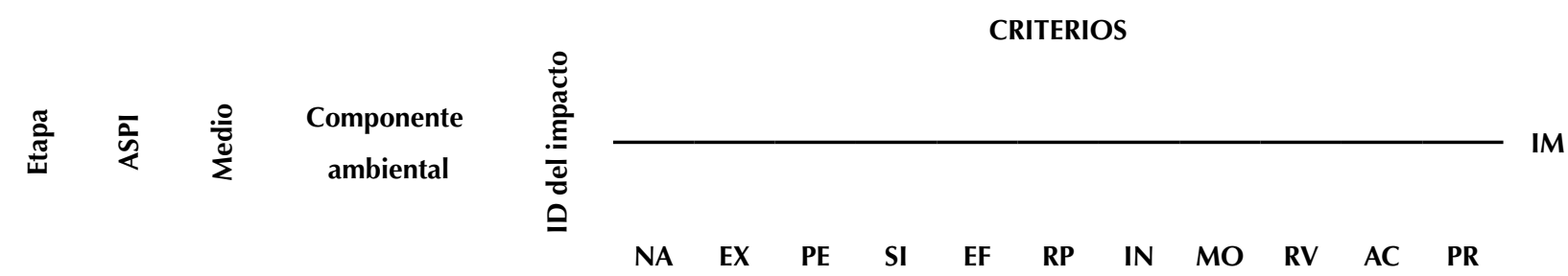

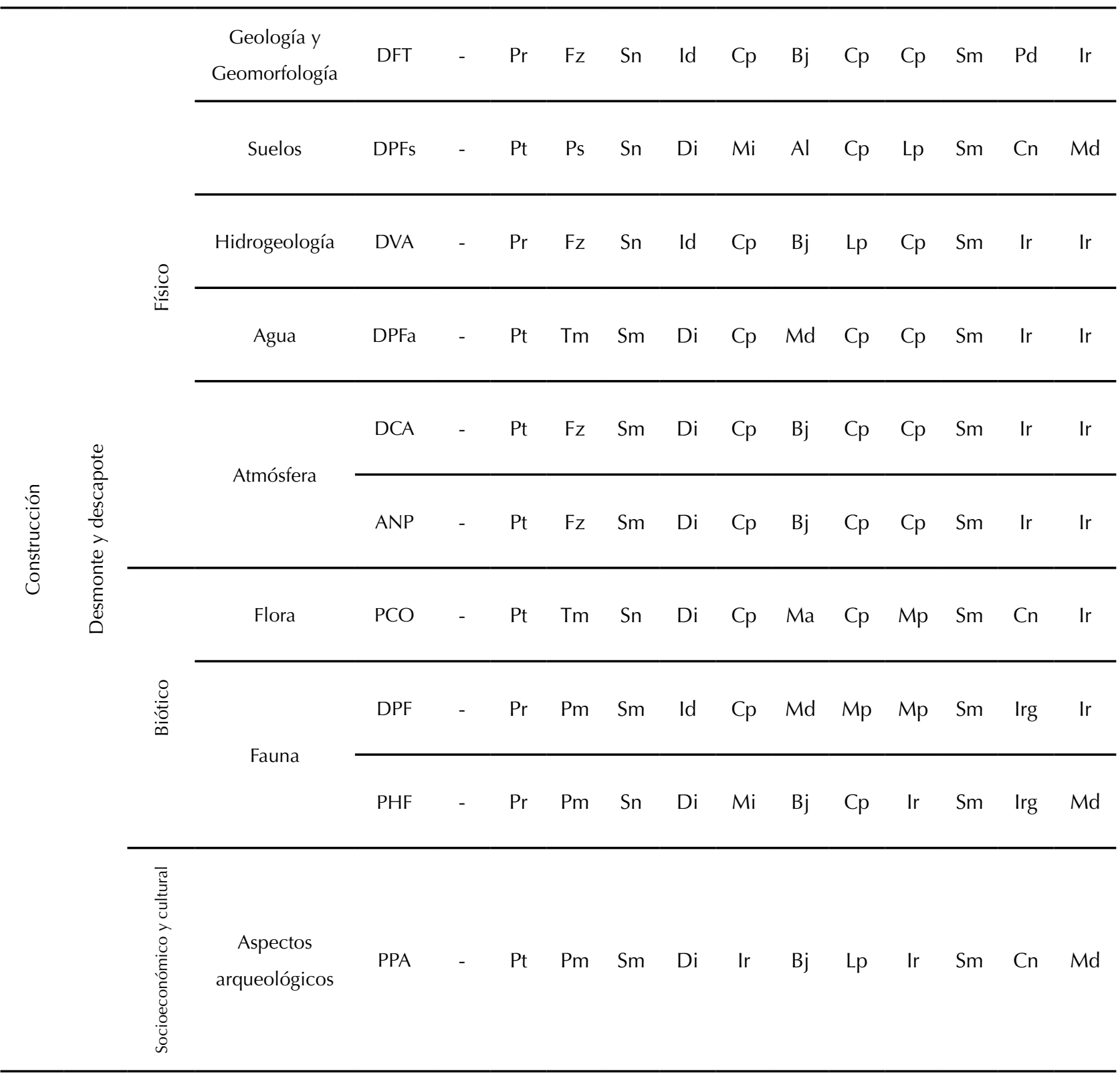

Fuente: Luque y Ramírez (2016). 
Tabla 4. Construcción de conjuntos difusos para variables de tipo cualitativas

\begin{tabular}{cc}
\hline CONSTRUCCIÓN NÚMEROS DIFUSOS TRAPEZOIDALES \\
\hline $\begin{array}{c}\text { CDi }(\mathrm{a} 1, \mathrm{a} 2, \mathrm{a} 3, \mathrm{a} 4), \mathrm{i}=1,2,3,4,5 \ldots \mathrm{p} \\
\text { Siendo } \mathrm{p} \text { el número de etiquetas por conjunto }\end{array}$ \\
\hline$a_{i}=\left\{\begin{array}{l}0 ; i=1 \\
(2 i-3) \Delta ; i \neq 1\end{array}\right\}$ & $b_{i}=\left\{\begin{array}{l}0 ; i=1 \\
(2 i-2) \Delta ; i \neq 1\end{array}\right\}$ \\
\hline$c_{i}=\left\{\begin{array}{l}(2 i-1) \Delta ; i \neq p \\
1 ; i=p\end{array}\right\}$ & $d_{i}=\left\{\begin{array}{l}(2 i) \Delta ; i \neq p \\
1 ; i=p\end{array}\right\}$ \\
\hline$\Delta=\frac{1}{2 p-1}$ & \\
\hline
\end{tabular}

Fuente: Luque y Ramírez (2016).

Una vez hecho lo anterior, se procedió a llevar los años a una escala de [0 - 1], lo cual permitió tener una mayor fiabilidad a la hora de realizar las operaciones entre los demás criterios definidos sobre el mismo universo de discurso (tabla 5).

Luego en la construcción de los conjuntos difusos se definieron los $\alpha$-cortes, y de esta manera se consiguió que los puntos de cruce o el punto superior de la intersección entre los subconjuntos difusos fueran 0,5 . A su vez, se tuvo en cuenta que el $25 \%$ de los datos de la etiqueta a corto plazo, que es la más pequeña en comparación a la etiqueta a mediano plazo, están representados por el valor 0,01, el cual se restó y se adhirió según la necesidad del subconjunto.

Para la etiqueta a mediano plazo y a largo plazo el menor rango de intervalo se dio en a mediano plazo, y el $25 \%$ equivale a 0,09.

Para el primer número trapezoidal [0,00,00,03 0,05] correspondiente al subconjunto a corto plazo los $\alpha$-cortes se definieron como $(0,(0,05-0,02 \alpha))$, en donde un $\alpha=0,5$ representa un intervalo de $(0$, $(0,04))$ evidenciando que su punto de cruce con el subconjunto a mediano plazo es al año uno (tabla 5).
De igual manera, al definir el $\alpha$-cortes para el subconjunto a mediano plazo se obtiene $((0,03+0,02 \alpha,(0,49-0,18 \alpha))$, en donde un $\alpha=0,5$ define el intervalo $((0,04),(0,4))$ evidenciando que los puntos de cruce con los subconjuntos a corto plazo y a largo plazo son en 1 y 10 años, respectivamente (tabla 1 ). Una mejor forma de observar estos datos para el criterio MO se muestran en la figura 2, cuyos rangos en años corresponden a:

- A corto plazo: [0,0 0,0 0,75 1,25].

- A mediano plazo: $[0,75 \quad 1,25 \quad 6,5$ $13,5]$.

- A largo plazo: $[6,5$ 13,5 1,0 1,0].

Por su parte, los conjuntos difusos para los criterios PE, RV y RP fueron creados de la misma forma, pero en este caso el límite superior se fijó en 30 años. Así, la construcción de los subconjuntos difusos asociados a los criterios de evaluación, en donde para cada etiqueta se representa por un número difuso trapezoidal se presentan en la tabla 1.

Finalmente, en términos de aplicar la metodología propuesta, se calculó la importancia difusa y se tomaron como 
Tabla 5. Rangos y escala de $[0,1]$ para las etiquetas a corto, mediano y largo plazo del criterio MO

\begin{tabular}{ccc}
\hline Etiquetas & Rangos & Escala de [0,1] \\
\hline A corto plazo & 0 años $\geq \mathrm{x} \leq 1$ año & $0 \leq$ años $\mathrm{x} \leq 0,04$ años \\
\hline A mediano plazo & 1 año $<\mathrm{x} \leq 10$ años & 0,04 años $<\mathrm{x} \leq 0,4$ años \\
\hline A largo plazo & 10 años $<\mathrm{x} \leq 25$ años & 0,4 años $<\mathrm{x} \leq 1$ año \\
\hline \multirow{2}{*}{$\operatorname{cons}(x, y)=\sup _{u \in U}\left(\min \left(\mu_{x}(u), \mu_{y}(u)\right)\right.$} & $(2)$
\end{tabular}

datos de partida la valoración de los criterios para los impactos generados por el proyecto.

Donde la importancia de una acción $(A j)$ sobre un factor $(F i)$ se determinó mediante el cálculo del valor de la consistencia (cons) entre el número difuso de la variable de salida de la fra, y la función de pertenencia de las variables definidas para el cálculo de la importancia (tabla 1). De este modo, la consistencia para dos funciones $(\mu x(u)) y(\mu y$ (u)), según Duarte (2000), se presenta en la ecuación (2):

La consistencia entre el conjunto difuso resultante del fra y cada una de las etiquetas de la variable de salida se interpretó como una medida de la posibilidad de que el resultado del fra tenga el significado semántico de la etiqueta lingüística correspondiente. Este valor numérico se clasificó en los siguientes intervalos:

- $\mathrm{Si}$ es mayor que 0,6666 es muy posiblemente.

- Si está entre 0,3333 y 0,6666 es posiblemente.

- Si es menor que 0,3333 es poco posiblemente.

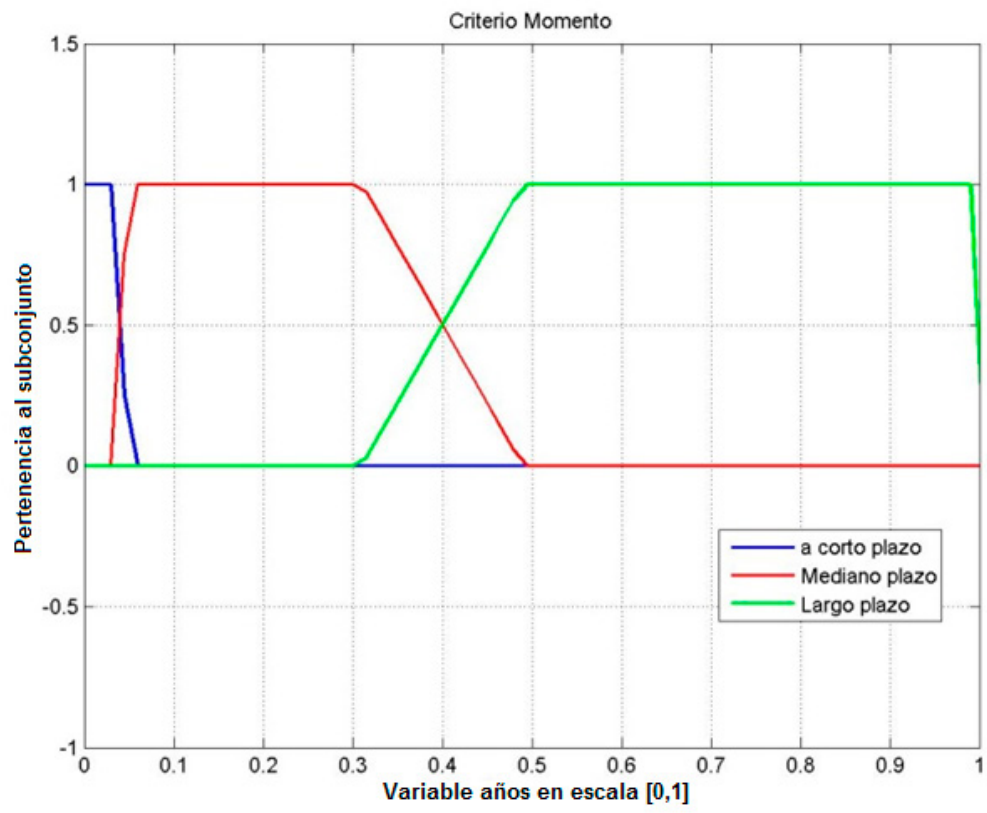

Figura 2. Ejemplo de la construcción del conjunto difuso para la variable momento Fuente: Luque y Ramírez (2016). 
- Si es igual a 0 no se califica la salida con la etiqueta.

Para evitar la entrada de la subjetividad nuevamente al momento de establecer la verdadera importancia del impacto, se escogió el valor de pertenencia que se encontraba más cerca del mayor criterio según la naturaleza del impacto; es decir, si la naturaleza era negativa, se escogía el criterio con algún grado de pertenencia más cercano a crítico, y si era de naturaleza positiva, el criterio con algún grado de pertenencia más cercano a significativo y se calificó su grado de pertenencia al criterio (figura 3).

En la tabla 6. se presentan los resultados de la valoración difusa del impacto en función de la importancia. Se encontró que estos, al incorporar los conjuntos difusos, se agrupaban en cuatro grandes categorías: muy posiblemente moderado, con 3 impactos; posiblemente severo, con 2 impactos; posiblemente moderado, con 3 impactos; y poco posiblemente severo, con 2 impactos.

\section{Validación de la EIA propuesta}

En la validación de las metodologías (Conesa vs. método de técnicas difusas) se debe partir de las categorías de valoración. Por ejemplo, la metodología convencional, si al calcular la importancia, esta se encuentra entre $-25 \leq$ I $\leq-50$, ubica los impactos en la categoría moderada, a pesar de estar al límite de la categoría severo $(-50<\mathrm{I} \leq-75)$, es decir, se encuentra excluido totalmente al no cumplir las reglas de pertenencia; mientras que la metodología difusa propuesta indica un grado de pertenencia entre el rango de $[0,1]$, lo cual hace que este sea catalogado como un impacto

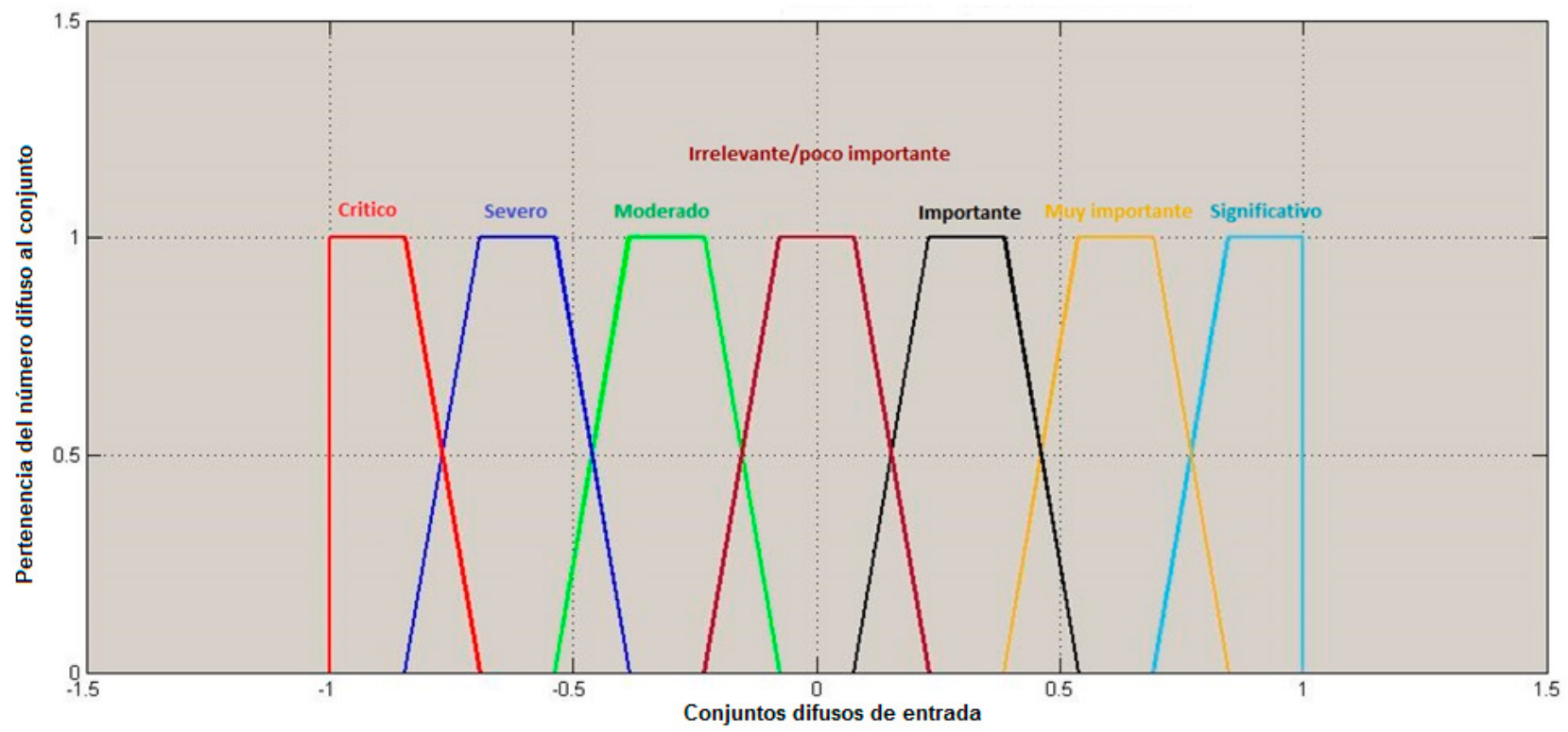

Figura 3. Importancia difusa de acuerdo con la metodología aplicada Fuente: Luque y Ramírez (2016) 
severo. Esta situación es fundamental para disminuir la subjetividad.

Otro aspecto importante es que con los resultados de la metodología propuesta (tabla 6), de los 10 impactos evaluados
$40 \%$ de ellos resultaron ser severos (poco posiblemente y posiblemente), y el porcentaje restante, moderados (posiblemente y muy posiblemente), mientras que con la metodología de Conesa

Tabla 6. Valoración difusa del impacto en función de la importancia.

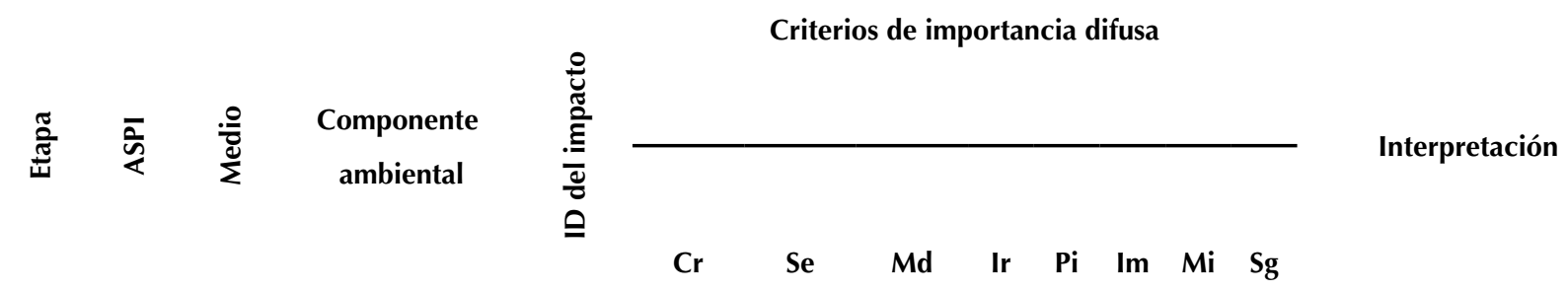

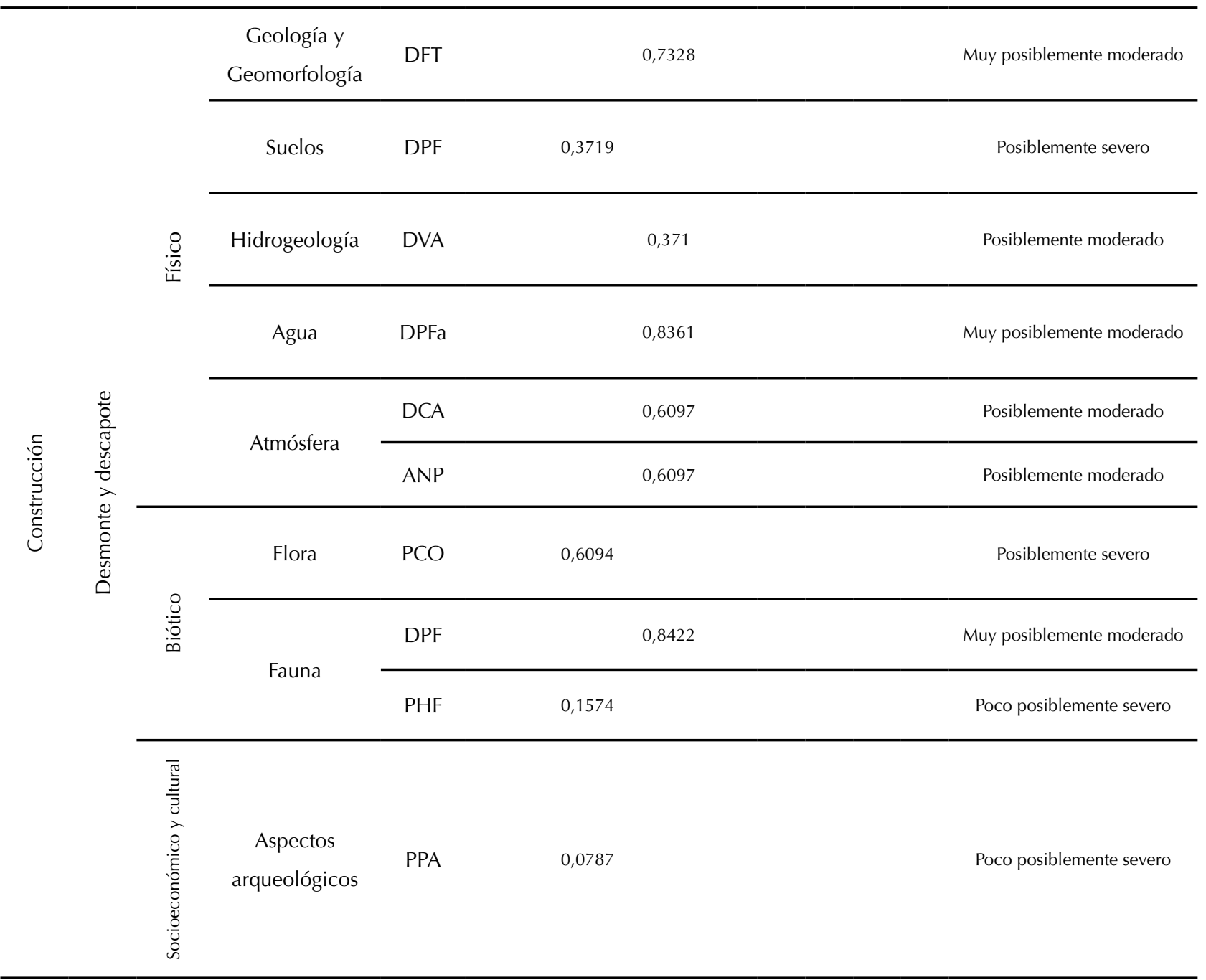

Fuente: Luque y Ramírez (2016)

Tecnura • p-ISSN: 0123-921X • e-ISSN: 2248-7638 • Vol. 24 No. $64 \bullet$ Abril - Junio de $2020 \bullet$ pp 48-65 
(tabla 3) no se presentan impactos severos; los impactos moderados representaron el 30 $\%$, y en su gran mayoría, fueron valorados como irrelevantes. Esto indica que Conesa es una metodología conservadora al momento de la valoración, como lo reconocen Toro, Martínez y Arrieta (2013) quienes encontraron que el método más utilizado es el cualitativo propuesto por Conesa, con modificaciones que disminuyen la efectividad de la EIA, y potencializan la subjetividad y el sesgo del evaluador.

Caso contrario ocurre con la metodología propuesta, que al ser más rigurosa y permitir un menor grado de subjetividad, hace que la importancia del impacto se resalte, sea este de naturaleza positiva o negativa.

Tabla 7. Ejemplo de las categorías de valoración entre las metodologías convencional y difusa

\begin{tabular}{l|c}
\hline \multicolumn{1}{c|}{ Metodología convencional } & Metodología difusa \\
\hline Moderado (-50) & Poco posiblemente irrelevante $(0,1257)$ \\
& Muy posiblemente moderado $(1,0)$ \\
& Muy posiblemente severo $(0,7189)$ \\
\hline
\end{tabular}

Fuente: Luque y Ramírez (2016)

Un claro ejemplo de cómo aumenta la exactitud de los resultados se da en el impacto pérdida de hábitat para fauna terrestre. En este mismo sentido, Viloria, Cadavid y Awad (2018) manifiestan que en Colombia se sigue evidenciando poca calidad de los estudios ambientales, una gran diversidad de métodos con inconsistencias metodológicas e incluso conceptuales, sumados al uso de juicios de valor que dependen del profesional que los ejecuta y a que las escalas de valoración cualitativas no son claras o adecuadas, lo cual sesga los resultados.

\section{CONCLUSIONES}

La metodología propuesta resalta las debilidades de las metodologías convencionales debido a la poca subjetividad involucrada al momento de dar valor a las variables de entrada, mientras que en la metodología convencional estos números van determinados por el usuario o por el método utilizado. Asimismo, las técnicas difusas se mantienen variantes únicamente en el número de etiquetas o subconjuntos del conjunto de partida, las cuales, a su vez, se establecen en un rango de pertenencia para cualquier valor sobre el universo de discurso.

La evaluación mediante técnicas difusas permitió la incorporación variables monótonas y continuas como por ejemplo resiliencia y cobertura, entre otras, las cuales se pudieron operar como un conjunto difuso. A su vez, se tiene como novedad el uso de la aritmética difusa 
mediante operaciones de intervalos definidos por los $\alpha$-cortes, los cuales facilitaron la ejecución de operaciones matemáticas para la obtención de la fra.

Esta metodología permitió modelar con una mayor exactitud la manera en que interactuará el proyecto y el ambiente, en comparación a las metodologías convencionales, lo cual ayudó a la oportuna incorporación de mejores medidas correctoras.

\section{Financiamiento}

La presente investigación fue financiada por los autores y formó parte del trabajo de pregrado en Ingeniería Ambiental en las Unidades Tecnológicas de Santander, de los ingenieros Germán Yesid Luque Díaz y Laura Cristina Ramírez Salinas.

\section{REFERENCIAS}

Arboleda, J.A. (2008). Manual para la evaluación ambiental de proyectos, obras o actividades. Medellín.

Avellaneda, A. (1990). Petróleo e impacto ambiental en Colombia. Revista de la Universidad Nacional (19441992), 6(24), 21-28.

Bailey, J. (1997). Environmental impact assessment and management: An underexplored relationship. Environmental Management, 21, 3-17. DOI: https://doi.org/https://doi. org/10.1007/s002679900032

Bear, D. (2003). Some modest suggestions for improving implementation of the National Environmental Policy Act. Natural Resources Journal, 43(4), 931-960. DOI: www.jstor.org/

\section{stable/24888893}

Bravo, E. (2005). Los impactos de la explotación petrolera en América Latina. Biodivesidad, 43, 1-9.

Collazos, A., Esquivel, C.L. y Paz, A. (2019). De los hidrocarburos a las energías renovables en Colombia. Cultura Latinoamericana, 29(1), 138-162. DOI: https://doi. org/http://dx.doi.org/10.14718/ CulturaLatinoam.2019.29.1.6

Corficolombiana (2018). Perspectivas económicas Corficolombiana. Proyecciones 2019: atención al volante. Bogotá.

Correa, C.A., Marulanda, G.A. y Panesso, A.F. (2016). Impacto de la penetración de la energía solar fotovoltaica en sistemas de distribución: estudio bajo supuestos del contexto colombiano. Tecnura, 20(50), 85-95. DOI: https:// doi.org/10.14483/udistrital.jour. tecnura.2016.4.a061

Duarte, O.G. (2000). Técnicas difusas en evaluación de impacto ambiental. [Tesis doctoral]. Universidad de Granada. Granada España. Recuperado de http://decsai.ugr.es/Documentos/ tesis_dpto/49.pdf

Duarte, O.G., Requena, I. y Rosario, Y. (2007). Fuzzy techniques for environmental-impact assessment in the mineral deposit of Punta Gorda (Moa, Cuba). Environmental Technology, 28(6), 659-669. DOI: https://doi. org/10.1080/09593332808618826

Dutta, P., Boruah, H. y Ali, T. (2011). Fuzzy Arithmetic with and without using $\alpha$-cut method: A comparative Study. International Journal of Latest Trends in Computing, 2(1), 99-107. 
Guerrero, M.E. (2018). Ruptura de oleoductos por interferencia externa, daño ambiental y sostenibilidad en Colombia. Revista Producción + Limpia, 13(2), 7-13.

Lozano, C. (2014). Diseño de una metodología de evaluación cuantitativa de impactos ambientales para pozos de perforación exploratoria del sector hidrocarburos. Bucaramanga: Unidades Tecnológicas de Santander.

Luque, G. y Ramírez, L. (2016). Técnicas difusas para la evaluación de impactos ambientales en el transporte de hidrocarburos en Colombia. Bucaramanga: Unidades Tecnológicas de Santander.

Luther, L. (2008). The national environmental policy act: Background and implementation. Washington D.C.: Congressional Research Service.

Malagón, J., Montoya, G. y Ruiz, C. (2016). La competitividad del sector de hidrocarburos en las diferentes regiones de Colombia. Programa de las Naciones Unidas para el Desarrollo (PNUD).

Marchevsky, N.J., Giubergia, A.A. y Ponce, N. H. (2018). Evaluación de impacto ambiental de la cantera "La Represa" en la provincia de San Luis, Argentina. Tecnura, 22(56), 51 61. DOI: https://doi.org/https://doi. org/10.14483/22487638.12907

Naciones Unidas. (2012). Informe del Consejo de Administración/Foro Ambiental Mundial a Nivel Ministerial. Nueva York. Recuperado de https:// undocs.org/pdf?symbol=es/A/67/25

Parada, E.A., Illera, M.J., Sepúlveda, S.B., Guevara, D. y Medina, B.
(2016). Sistema de control domótico de bajo costo: un respaldo a la generación ecológica de energía eléctrica en Colombia. Tecnura, 20(49), 120 132. DOI: https://doi.org/10.14483/ udistrital.jour.tecnura.2016.3.a08

Poveda, G. Y Mesa, O. (1995). Efectos hidrológicos de la deforestación. Energética, 16, 91-102.

Rojas, A., Ducuara, Y. y Moreno, R. (2015). Escenarios energéticos a 2050 con integración de fuentes de energía eléctrica renovables en Colombia. Tecnura, 19(CITIE), 83-89. DOI: https://doi.org/http://dx.doi. org/10.14483/udistrital.jour.tecnura.2015.ICE.a10

Toro, J., Martínez, R. y Arrieta, G. (2013). Métodos de evaluación de impacto ambiental en Colombia. Revista de Investigación Agraria y Ambiental, $4(2), 43-53$.

Toro, J., Requena, I. y Zamorano, M. (2010). Environmental impact assessment in Colombia: critical analysis and proposals for improvement. Environmental Impact Assessment Review, 30(4), 247-261.

Toro, J., Duarte, O., Requena, I. y Zamorano, M. (2012). Determining vulnerability importance in environmental impact assessment. The case of Colombia. Environmental Impact Assessment Review, 32(1), 107-117.

Villareal, E. y Arango, D. (2014). Estrategias para el entrenamiento de redes neuronales de números difuso. Tecnura, 18(40), 36-47. DOI: https:// doi.org/https://doi.org/10.14483/udistrital.jour.tecnura.2014.2.a03 
Viloria, M.I., Cadavid, L. y Awad, G. (2018). Metodología para evaluación de impacto ambiental de proyectos de infraestructura en Colombia. Ciencia e Ingeniería Neogranadina, 28(2), 121-156. DOI: https://doi.org/ https://doi.org/10.18359/rcin.2941

Zadeh, L.A. (1975). The concept of a linguistic variable and its application to approximate reasoning-l. Information Sciences, 8(3), 199249. DOI: https://doi.org/https://doi. org/10.1016/0020-0255(75)90036-5

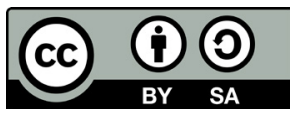

